\title{
Management Competencies in the Health Sector: Case of Zimbabwean Doctors in Matabeleland South Province
}

\author{
Cleopatra Matanhire-Zihanzu* Joel Penn Ndlovu \\ Graduate School of Business, Faculty of Commerce, National University of Science \& Technology, \\ PO Box AC 939, Ascot, Bulawayo, Zimbabwe
}

\begin{abstract}
The Zimbabwean Health Sector had commendable management and was a top performer in the African Continent. In the mid 1990's the country experienced a socio-economic collapse resulting in deterioration of the national health system. The weakening of the Zimbabwean health sector has mainly been attributed to the poor economic performance in the past two decades, however the contribution of management to the problem has not been analyzed. In the Zimbabwean context, all doctors have some management responsibilities that they are expected to deliver, because of shortage of the skilled health workers, often clinicians end up running medical institutions even in interim capacity. The aim of this study was to investigate current management competencies in the Zimbabwean health sector.An online questionnaire was used to assess the management competencies of 28 medical and dental practitioners in Matebeleland South Province. Furthermore, interviews were conducted with key informants in varying levels of the Ministry of Health and Child Care (MOHCC) Human Resource departments. Research findings revealed that most medical and dental practitioners have poor management competencies in running health institutions due to lack of training in management and administration in their undergraduate medical training and compromised various human resource processes. On this basis, it is recommended that training in management be incorporated into undergraduate medical training curriculums, the medical and dental practitioners' council to work with professional bodies to implement current business management guidelines and the Health Service Board to collaborate with the Public Service Commission to revive management and administrative training programs for the MOHCC.
\end{abstract}

Keywords: Management competencies, medical and dental practitioners, human resources, health management

DOI: $10.7176 / \mathrm{EJBM} / 12-14-05$

Publication date:May $31^{\text {st }} 2020$

\section{Introduction}

\subsection{Background}

The National Health Strategy 2016-2020 (2015,p3) informs that following independence in 1980, the Zimbabwean Health delivery system was robust, there were high quality clinical services and there was effective coverage of medical interventions. Zimbabwe was a top performer in the African Continent and management in the sector was commendable. In the mid 1990's the country experienced a socio-economic collapse resulting in deformation of the national health system, there was a decline in key health indicators and public health service availability

LaGrone et al. (2017) stresses that government funding for the health sector reduced significantly which resulted in low and declined pay for health workers. High attrition of experienced managers compromised provision of supportive supervision and performance monitoring of health workers including medical doctors. Loss of senior staff resulted in less experienced staff assuming positions they were ill equipped for, this also meant there were few remaining mentors to provide on the job training.

According to Haley et al. (2017), eighty percent of medical graduates left for neighboring overseas countries for better employment opportunities. Several medical schools and training facilities closed due to budget constraints and hence there was a reduction in availability of educational and training opportunities in Zimbabwe. World Health Organization (2018) affirms substantial outmigration, transfers to the private sector and non-health fields, early retirement, and death, all contributed to health workforce attrition and was compounded by a low replacement rate.

Witter et al. (2017) notes that the only medical school in the country lost seventy percent of its theory staff complement. Vacant academic posts at medical schools and tutor posts, outdated training materials and curricula, lack of tools and equipment to guide treatment at the point of care contributed to poor qualification among graduating health professionals. The quality of medical graduates was compromised, the health institutions they ran deteriorated in standards. The nation had an understaffed and overburdened health workforce which resulted in mismanagement of the health sector.

Doctors are both managers and are managed. "All practicing doctors are responsible for the use of resources; many will also lead teams or be involved in the supervision of colleagues and most will work in management systems, whether in the public sector or in the private sector. Doctors have responsibilities to their patients, employers and those who contract their services." (Business management responsibility guidelines for Medical and Dental Practitioners, 2011). In the Ministry of Health and Child Care (MOHCC) Job Description for Medical 
doctors in government institutions (1995) it is expressed that doctors management roles often involve responsibility for teams, people and the resources they use as they carry out supervision, administration, medical audits and teach junior staff.

Wong et al. (2015) identify the lack of information and poor management of health care systems and institutions as key factors which deter progress in the health sector

\subsection{The Health Sector in Zimbabwe}

Mugwagwa et al. (2017) describes the Zimbabwe health sector as comprising of a public and private health system. The building blocks of the health system in Zimbabwe whether public or private comprise of service delivery, the health workforce, information, financing, leadership (also referred to as governance) and various medical products. The Ministry of Health and Child Care (MOHCC) develops policy and provides overall guidance to the national health system.

The Zimbabwe National Health Financing Strategy (2017) explains the public health system is run by the Ministry of Health and Child Care (MOHCC) and provides the largest health care services. Health care is delivered through 1,848 facilities, most of which are public health care facilities. The health services are delivered at primary, secondary, tertiary, and quaternary levels. The system is complemented by mission hospitals and receives healthcare delivery support from several nongovernmental organizations (NGO). It is responsible for functions such as the determination of funding allocation, policy and administrative guidance, approvals of staff hire at the district and provincial levels.

MOHCC (2017) further elaborates on the provincial and district level structure. The Provincial Medical Directorate (PMD) office administers provincial and all district health facilities within its province; its function is to make certain that the province's health services meet the needs of the population, as well as MOHCC objectives, goals, and health policies. A Provincial Health Executive provides direct oversight to provincial hospitals. The PMD is also responsible for allocating Government of Zimbabwe funds to the provincial hospitals and district health offices. At the district level, District Health Officers (DHOs) have responsibilities similar to their provincial level- counterparts, except that they play a more direct role in administering and managing rural health clinics (the lowest level of primary care facilities), as rural health facilities may only have a nurse on staff to provide primary care services and no administrative staff. District hospitals are overseen by a District Health Executive. A district health council provides oversight to the district health office. Rural Health Clinics are also overseen by a Rural District Council. PMDs and DHOs are key management representatives of the MOHCC.

MOHCC Client Service Charter (2008) identifies the health sector as a service industry which has clients and stakeholders that it depends on to be operational. The MOHCC divisions, directorate and medical institutions have medical doctors leading and running the show, working in conjunction with key clients and stakeholders of the health sector. Figure 1 shows the Structure of the Public Health System in the Ministry of Health and Child Welfare Zimbabwe (2013). The various responsibilities in their diversity and vastness require good management skills and practices for the public health sector to thrive.

Mugwagwa et al. (2017) broadly describes the private health sector as part of a country's economic system that is owned, controlled, and run by private entities for profit making reasons or privately owned but for nonprofit organizations. MOHCC indicates there are 101 private health facilities and 87 mission facilities offering primary and secondary health care and 36 medical insurance companies in Zimbabwe. The structure and composition of private health organizations are multiple and vary according to institution and the purpose it serves.

\section{Methodology}

A mixed methods approach was adopted in this study as it was more appropriate in studying the nature of the effects identified and the consequences of the implementation. The population for this study comprised of all medical and dental practitioners in Matabeleland South Province. The key informants included human resource personnel from the Ministry of Health and Child Care and Matabeleland South Province. The sample size of the population was 35 respondents who comprised of 5 key informants and 30 respondents for the questionnaire. An online questionnaire was issued to the 30 respondents. In addition to these primary methods, secondary sources such as literature review, newspapers, observation, journals, and internet research were utilized in this research to have a credible data collection system.

\section{Results}

Out of the 30 respondents administered with the questionnaire, 28 respondents representing $93 \%$ of respondents answered. The high response rate might be attributed to the effectiveness of administering the questionnaire through a link sent on social media platforms as well as a bright and interactive response page with clear and precise questions that enabled respondents to complete the questionnaire in a few minutes. The study expected to conduct 5 interviews, however, the researcher managed to interview only 4 respondents, representing $80 \%$ of the targeted interviews while 1 was not available for interviews representing $20 \%$ of interview non-response rate. On 
the administered questionnaires $57.14 \%$ of respondents were female participants while $42.86 \%$ were male respondents. The results implied that female participants were more than male respondents in the medical fraternity in the Matabeleland South province. However, information from interviews indicate that the medical fraternity is dominated mainly by male doctors.

Table 1: Number of Years in Medical Practice

\begin{tabular}{|l|r|r|r|}
\hline \multicolumn{1}{|c|}{ Answer Choices } & \multicolumn{2}{|c|}{ Frequencies } \\
\hline $\mathbf{1 - 5}$ & & $89.29 \%$ & 25 \\
\hline $\mathbf{6 - 1 0}$ & & $7.14 \%$ & 2 \\
\hline $\mathbf{1 1 - 1 5}$ & & $0.00 \%$ & 0 \\
\hline$>16$ & Answered & $3.57 \%$ & 1 \\
\hline & Skipped & $\mathbf{2 8}$ \\
\hline
\end{tabular}

This data reveals that generally Matabeleland South Province has a young group of doctors in the medical fraternity.

Table 2: Perception of Current Management Practices

\begin{tabular}{|c|c|c|}
\hline Answer Choices & Frequencies & \\
\hline High & $21.43 \%$ & 6 \\
\hline Average & $53.57 \%$ & 15 \\
\hline Low & $25.00 \%$ & 7 \\
\hline \multirow[t]{3}{*}{ Do not Know } & $0.00 \%$ & 0 \\
\hline & Answered & 28 \\
\hline & Skipped & $\mathbf{0}$ \\
\hline
\end{tabular}

$53.57 \%$ of the respondents had average knowledge of management principles and practices, $21.43 \%$ had a high appreciation of management practices whilst $25 \%$ of the respondents had low appreciation of management practices. Interviews with HR officials indicated that most doctors in management positions start off with little appreciation of management practices, but they eventually increase on the job.

Figure 2: Previous Management Training History

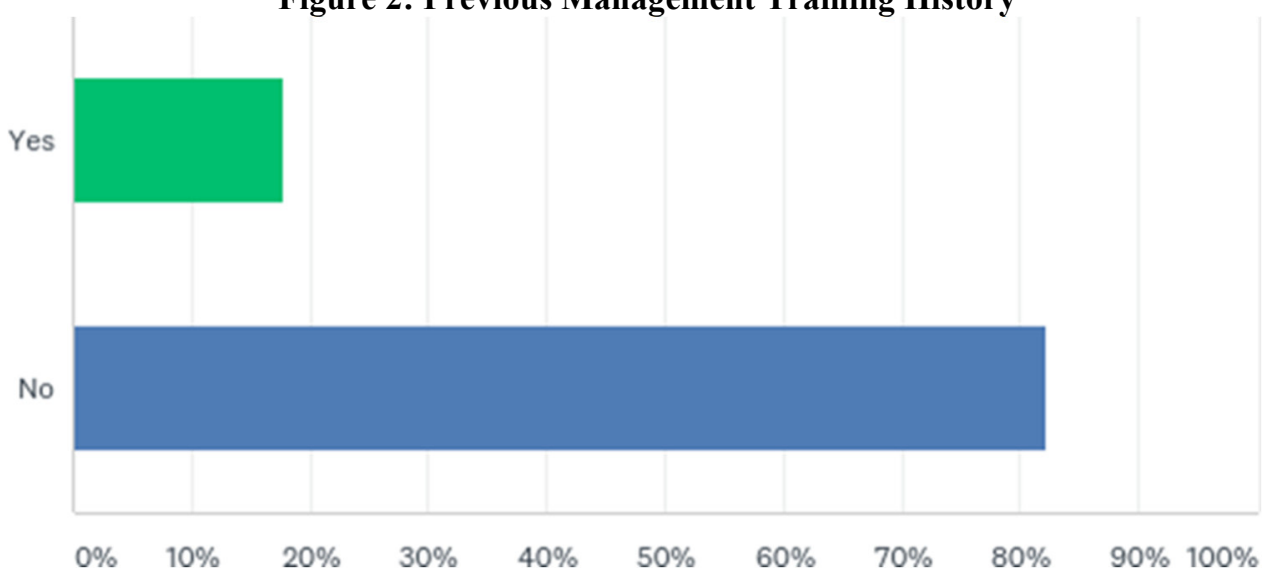

Figure 2 indicates that $82.14 \%$ of the respondents have no prior form of management training, whilst $17.86 \%$ of the respondents indicating that they have some form of management training history. This is consistent with Loh (2014)'s remarks that hospitals are complex organizations and so attention has to be given to the development of management competencies of the managers managing them.

Of the respondents that had indicated previously undergoing some form of management training, the average training duration for $40 \%$ was less than one week and another $40 \%$ indicated undergoing 1-6 weeks long training. $20 \%$ of the respondents underwent $2-18$-month long training. This data highlights that, while prior training may exist, the quality and effectiveness of this training, as evidenced by the duration, may not be enough. 


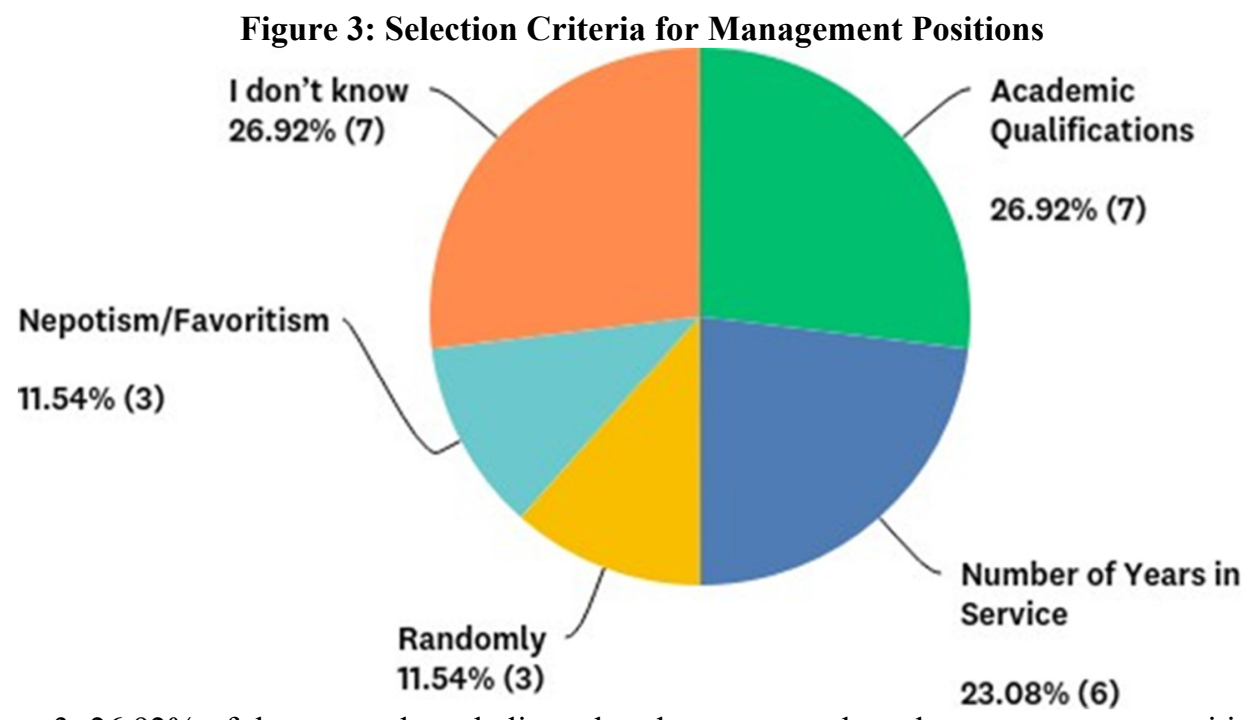

In Figure $3,26.92 \%$ of the respondents believe that doctors are selected to management positions based on their educational qualifications, another $26.92 \%$ are not aware of the selection criteria for management positions, $23.08 \%$ believe that selection is based on the number of years in service, $11.54 \%$ believe that the selection criteria are random and 11.54 believe that selection is based on nepotism and favoritism.

Regarding orientation on commencing a new job, $48 \%$ of the respondents underwent job orientation when they joined a health institution, $32 \%$ were not sure whether they received formal orientation and $20 \%$ did not receive any. The study also evaluated participants knowledge of their job descriptions, to which $79.17 \%$ of the respondents had knowledge, $16.67 \%$ not knowing their job description and $4.17 \%$ of the respondents unaware if they even have job descriptions. Knowledge of job descriptions is vital as it helps in improving performance when one is aware of what they are employed to do

Figure 4: In-Service Leadership/Management Training

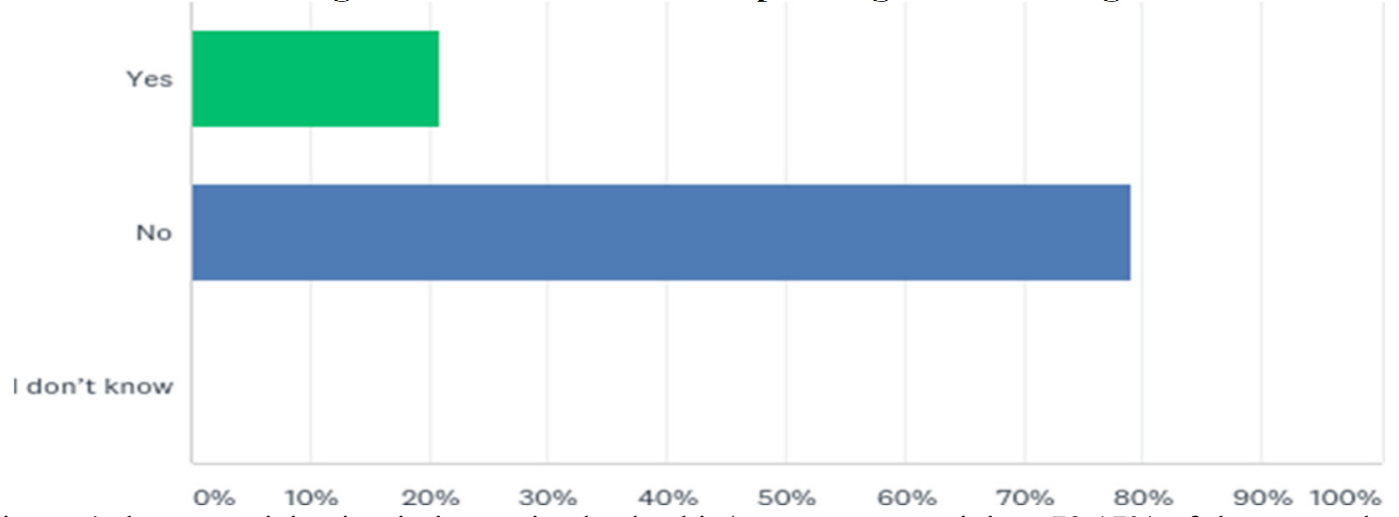

Figure 4 shows participation in in-service leadership/management training $.79 .17 \%$ of the respondents had never participated in any in-service management training course, whilst $20.83 \%$ had been involved in a form of management training course. The large proportion of doctors without participation in a management training course and the lack of any formal management training in medical school suggests that there is a significant deficiency in training of doctors in management. 
Figure 5: Importance for Doctors to Learn Management Principles

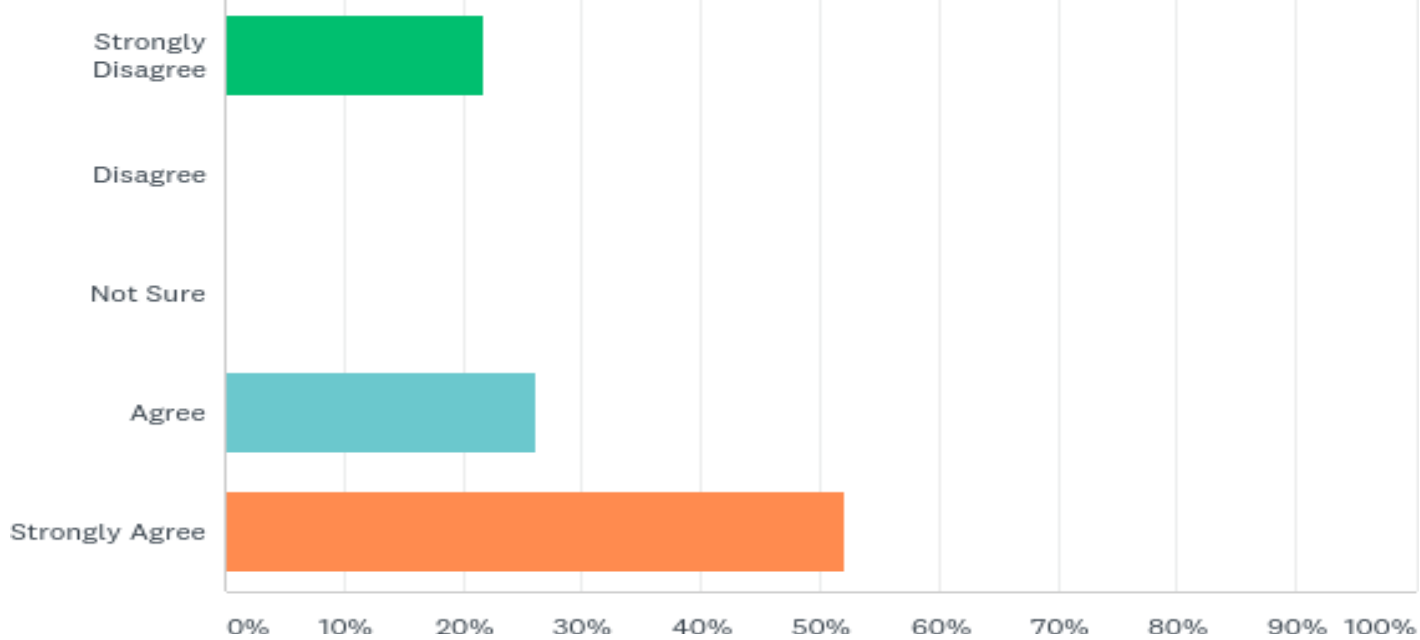

When asked ,'Is it important for Doctors to learn about Management Principles and a Module on Management should be included in Medical School?' $52.17 \%$ of the respondents strongly agreed that it was important for doctors to learn about management principles and that management courses should be introduced in Medical school, an additional $26.09 \%$ of the respondents agreed whilst $21.74 \%$ of the respondents strongly disagreed on doctors learning about management principles and the inclusion of management courses in medical school.

Table 3: A Doctor without formal training in management will make a poor manager

\begin{tabular}{|c|c|c|}
\hline Answer Choices & Frequencies & \\
\hline Strongly Disagree & $8.70 \%$ & 2 \\
\hline Disagree & $21.74 \%$ & 5 \\
\hline Not Sure & $17.39 \%$ & 4 \\
\hline Agree & $34.78 \%$ & 8 \\
\hline \multirow[t]{3}{*}{ Strongly Agree } & $17.39 \%$ & 4 \\
\hline & Answered & 23 \\
\hline & Skipped & 5 \\
\hline
\end{tabular}

Data in table 3 and Figure 5 indicates that $17.39 \%$ of the respondents strongly agree while $34.78 \%$ agree that a doctor without formal management training will become a poor manager. $21.74 \%$ of the respondents however disagree with a further $8.70 \%$ strongly disagreeing that without formal management training, a doctor will become a poor manager.

Table 4: Doctors Possess the Skills to Prepare Budgets, Manage Staff and Engage Stakeholders

\begin{tabular}{|c|c|c|}
\hline Answer Choices3.2 & Frequencies & \\
\hline Strongly Disagree & $8.70 \%$ & 2 \\
\hline Disagree & $21.74 \%$ & 5 \\
\hline Not Sure & $47.83 \%$ & 11 \\
\hline Agree & $21.74 \%$ & 5 \\
\hline \multirow[t]{3}{*}{ Strongly Agree } & $0.00 \%$ & 0 \\
\hline & Answered & 23 \\
\hline & Skipped & 5 \\
\hline
\end{tabular}

Data in Table 4 shows $21.74 \%$ of the respondents agree that doctors possess enough management skills while a similar proportion of $21.74 \%$ disagree and $47.83 \%$ of the respondents were not sure whether doctors possessed enough management skills to run a health institution.

\section{Discussion of Results}

\subsection{The current profiles of medical doctors in management positions}

Majority of study respondents possess a basic undergraduate degree in Medicine or Dentistry and have 5years or less years in medical practice. Most practitioners are engaged in dual practice of medicine in both public and private health sectors. Most Respondents indicated possessing an average knowledge of management and administration of health institutions. Only a minority of the study participants had received some form of management training. The trend shows foreign trained practitioners having received the training as part of undergraduate studies whilst Zimbabwean trained practitioners received it in in-service workshops of less than 1 week in duration and outliers in postgraduate studies.

The results implied that female participants were more than male respondents in the medical fraternity in the 
Matabeleland South province. However, information from interviews indicate that the medical fraternity is dominated mainly by male doctors. The results are therefore inconsistent with the literature reviewed that traditionally the roles of women were related to the family rather than the business sphere and that made them believe that they were not suitable for leadership roles. Women had been categorized as more likely to be working in the informal sector due to women often assume a balancing act of income earners, performing domestic chores and having primary responsibility of taking care of the elderly and the children (ILO, 2002). This can also be best explained by the fact that women also face discrimination when it comes to having access to education and training in the African society.

\subsection{To determine selection criterion of medical doctors in management positions}

The Selection criterion of medical and dental practitioners are basically adherent to choice of a candidate with the highest score on the template interview score-sheet that is issued by the Human Resources Department as standard protocol to each institution under the MOHCC, However each institution can make additional adjustments according to their preferences for a particular job opening. Most practitioners are required to perform some management and administrative duties, in the appointed junior medical or dental officer capacity. Appointment is based on fulfilment of the requirement of a basic undergraduate degree in Medicine or Dentistry. Re-grading to more senior positions in the medical or dental officer is done according to number of years' experience within the line of advancement. With regards to higher offices in the seven MOHCC directorates referred to in chapter one, the principle directors and deputy directors in the Ministry of Health and Child Care are required to have an additional Masters in Public Health qualification or equivalent Master's degree qualification in their field of focus for them to occupy office.

\subsection{To evaluate current management training protocols for medical doctors}

Prior to the year 2006, all executors of managerial /administrative duties in Ministry of Health institutions, including all medical and dental practitioners were required by the Public Service Commission (PSC) to attend refresher courses quarterly at institutions such as Zimbabwe Public Administrative and Management (ZIPAM) Institute, Elangeni Institute of Training and Development as well as the Highlands Institute of Training and Development Centre and others which had been established in the country by the PSC to equip the cadres with competencies required for their respective positions. However, in 2006 the Health Services Board was created to make the MOHCC handle its issues at an independent level due to the growth of the Ministry. Furthermore in 2009 with the establishment of the Government of National Unity (GNU) after the 2008 Harmonized elections, the new government froze most medical and dental practitioner posts which had been left unoccupied with the clinicians seeking employment in other countries in a bid to control a ballooning wage bill. Up until present day 2018 most of these posts have remained unoccupied as the resolution was never reversed. This saw inexperienced clinicians who were already in the system assuming administrative and management posts. The poor economic status of the country and advent of the Health Services Board have led to the demise of a once good management and administrative training program for employees by the Public Service Commission. At present there is few, if any training programs for employees when donor funding is availed, however most of the programs focus on the area of interest of the donor (mostly Non-governmental organizations) sponsoring and hence there is a definite neglect on the development of management and administrative competencies of the clinicians.

With regards to orientation, most questionnaire respondents indicated that they received orientation for their clinical duties only whilst a minority did not receive any orientation at all and did not know their full job description. Human Resource personnel from the head office, provincial and institution level indicated in their interviews that ideally the clinicians are supposed to receive orientation when they assume duty. The orientation is meant to be in various areas of their duties and responsibilities including in management and administration, however at present this is not adhered to. The only fail-safe existing is a six-month probation period that they undergo which is also not strictly adhered to if a practitioner fails to deliver on the duties. In the past there used to be orientation material of handbooks and manuals but when the Public Service Commission was replaced by the Health Services Board the system collapsed and was never fully revived. It is from these perspectives that the current management training protocols can be said to be of poor quality and not standardized.

\subsection{To appreciate medical doctors' perceptions on the performance versus expectations of their managerial roles}

The study revealed that both human resource personnel and medical doctors perceive there is poor management of health institutions by most practitioners as they lack the knowledge in Human resources, financial management, strategic planning and performance management which form a significant amount of their management and administrative work. Hence their performance is falling short of the expectations of what they are supposed to deliver 


\section{Conclusion}

Research findings show the majority of medical and dental have poor management competencies in running health institutions due to compromised various human resource processes and lack of training in management and administration in their undergraduate medical training. While doctors concede that they do not receive formal management training, they still get the opportunity to lead as managers and administrators in health institutions. However, the is a great deficiency of management skills in doctors who are managers, and this has a significant bearing on the performance of the health institutions.

Recommendations suggested are there should be an introduction of management courses in undergraduate medical degree programs as some foreign universities have done in their education curriculums, deliberate training programs have to be conducted in the work place so as to equip the medical and dental practitioners with the relevant management competencies for optimum performance in management duties and roles and aim of retaining the skills and knowledge, The Health Services Board is to investigate and review the current performance appraisal and management protocols as there is a clear misunderstanding of how the current system operates and the system is not performing the function that it should serve. Furthermore, refresher courses should be reintroduced utilizing existing resources such as the Public Service Commission infrastructure and internal training personnel. The introduction of a collaborative effort between the two existing entities can help the health sector transition into an improved status of management which would be a long-term investment which cannot be replaced overnight. The Medical and Dental Practitioners Council (MDPCZ) is to work with professional bodies such as the Zimbabwe Medical Association (ZIMA) and Zimbabwe Dental Association (ZIDA) in implementation of their existing Business management responsibility guidelines to be incorporated into Continuous medical education or continuous professional development programs that are conducted annually for practitioners to have their medical practicing licenses renewed.

\section{References}

Haley, C.A., Vermund, S.H., Moyo, P. et al. (2017), 'Impact of a critical health workforce shortage on child health in Zimbabwe: a country case study of progress in child survival, 2000- 2013.' Health Policy and planning, Vol. 32 Iss:5, pp.613-624

LaGrone, L.N, Ticker L.N., Egoavi, 1. H., Matte, J.J., et al. (2017) 'Surgery and Trauma care providers perception of the impact of dual practice employment on quality of care provider in an Andean country.' BJS Vol.104 Iss:6, pp.704-709

Loh, E. (2014b), "LAMP: leadership, advocate, management, professional - a new simple and dynamic medical management competency model for doctors", Gibaran Journal of Applied Management, Vol. 6 No. 2, pp. 6486.

Ministry of Health and Child Care. (2015) National Health Strategy 2016 - 2020 (2015). pp.3-6

Medical and Dental Practitioners Council of Zimbabwe. (2016) Policy

on the Administration of Health Institutions. pp.3-6

Medical and Dental Practitioners Council. (2011) Business management

responsibility guidelines for Medical and Dental Practitioners.p.2

Ministry of Health and Child Care. (2017) Zimbabwe Health Financing Strategy 2017, pp.1-7

Ministry of Health and Child Care. (1995) Job Description for Medical doctors in government institutions.pp.7-10

Ministry of Health and Child Care. (2008) Client Service Charter.pp.3-4

Mugwagwa, J.T., Chinyadza, J.K, Banda, G. (2017) 'Private sector participation in healthcare in Zimbabwe: What's the value added?' J Health c Communi.Vol.2. Iss.2. Available from: http doi

: 10.4172/2472-1654.1000 50 [Accessed on16 April 2018]

Witter, S., Wurie, H., Chandiwana, P, et al. (2017) 'How do health workers experience and cope with shades? Learning from four fragile and conflict affected health systems in Uganda, Sierra Leone, Zimbabwe and Cathodia.' Health Policy and planning. Vol.32, Iss:3, pp. iii3 - iiii13.

Wong, Simon,C. K., \& Patrick, C. L. (2017) 'Competencies of training professionals in the Hong Kong hotel industry,' Journal of Human Resources in Hospitality \& Tourism.Vol.16.Iss.4.pp.384-400 Available from: https://doi.org/10.1080/15332845.2017.1266872. Accessed: 22 April 2018

Wong, E. L. Y, Yeah, E, Chau, P.Y.K. Yam, C.H.K., Cheung, A.W.L. Fung, H. (2015) 'How shall we examine and learn about public private partnerships (PPPs) in the health sector? Realist evaluation of PPPs in Hong Kong.' Social Science and Medicine.Iss.147, pp.261- 263 


\section{Notes}

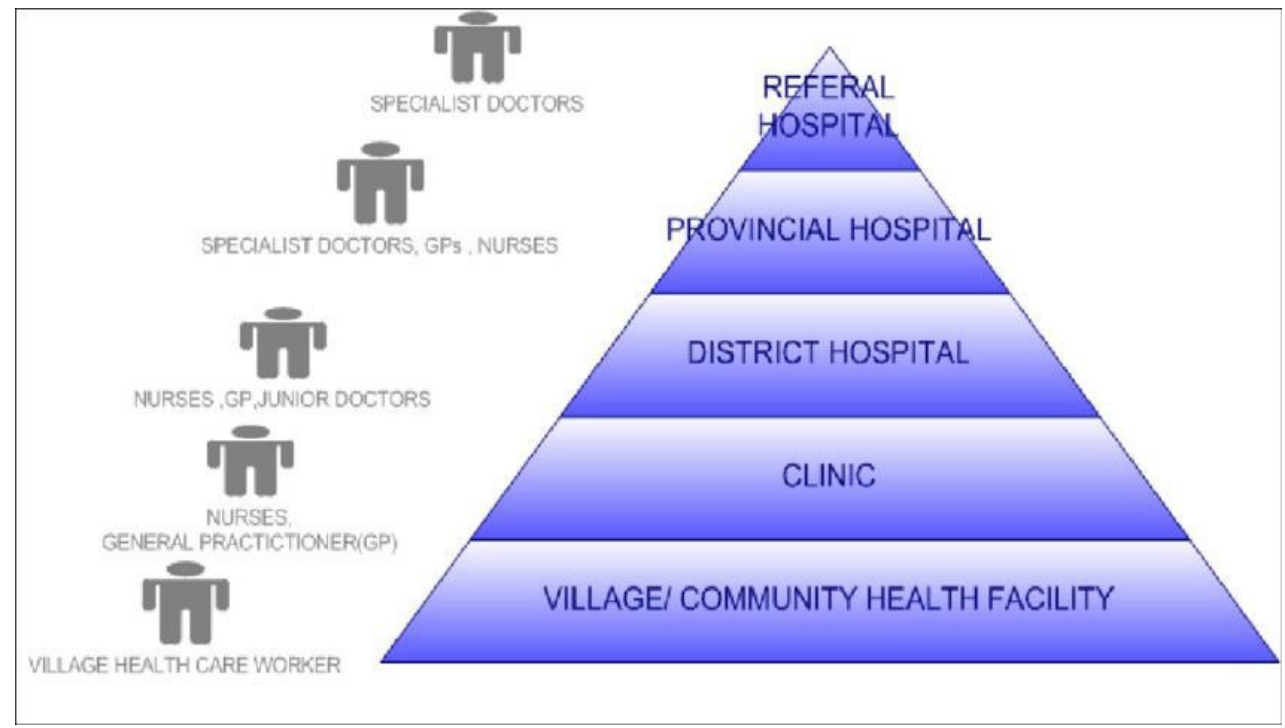

Figure 1. Structure of the Public Health System in Zimbabwe (2013)

Cleopatra Matanhire-Zihanzu became a member of the Zimbabwe Dental Association (ZIDA) in 2015, a member of the Zimbabwe Women Doctors Association (ZWDA) in 2019 and a Mandela Washington Fellow in the Young African Leaders Initiative in 2017.

Cleopatra was born in Harare, Zimbabwe. She holds a master's in business administration Degree from the National University of Science and Technology, Bulawayo, Zimbabwe,2018. Cleopatra has a bachelor's degree in Dental Surgery from the University of Zimbabwe, Harare, Zimbabwe, 2015. She also holds a certificate in Civic Leadership from Staley School of Leadership Studies, Kansas State University, Manhattan Kansas, United States of America, 2017. She is an advocate for health equity and Oral Health, she majors in research and management of health systems.

Joel P. Ndlovu became a member of Faculty at Bulawayo Polytechnic in 2002, and a member of Graduate School of Business, NUST, 2005. He was born in Nkayi on 20/07/1956, BA Double major in business administration and Bible, Daystar University, Nairobi, Kenya, 1992. He holds an MA in Biblical Studies, Nairobi International School of Theology, Nairobi, Kenya, 1994 and an MBA, NUST, Bulawayo, Zimbabwe, 2001. 